\title{
Design of Oil and Gas Industry Pipeline Systems Taking into Account Out-of-Roundness of Pipes and Change of Transportation Mode
}

\author{
Bakhtizin Ramil Nazifovich \\ Ufa State Petroleum Technological University, \\ the Republic of Bashkortostan, Ufa city, Russia \\ rektor@ rusoil.net
}

\section{Azmetov Khasan Akhmetzievich}

Ufa State Petroleum Technological University, the Republic of Bashkortostan, Ufa city, Russia zpavlova@mail.ru

\author{
Pavlova Zukhra Khasanovna \\ Ufa State Petroleum Technological University, \\ the Republic of Bashkortostan, Ufa city, Russia \\ zpavlova@mail.ru \\ Makarenko Oleg Anatolyevich \\ Ufa State Petroleum Technological University, \\ the Republic of Bashkortostan, Ufa city, Russia \\ akvamakoleg@mail.ru
}

\begin{abstract}
Major requirement to the design of oil and gas industry pipeline systems is the provision of their safety under actual operating conditions taking into account the actual technical properties of the used items. Experience shows that the actual operating conditions of pipeline system objects are characterized by the deviations from the accepted conditions at the design stage. The main cause of deviation of the actual operating conditions from the accepted ones is the change of transportation mode before the complete pipeline shutdown. Items used for construction of pipeline systems, including pipelines, have various manufacturing defects within the requirements of the current regulatory documents. Specified deviations and manufacturing defects lead to the increase in the level of mechanical stresses in load-bearing members of structures and decreasing of safety of pipeline systems operation. This stipulates the necessity of performing analysis of influence of the technological operating parameters and pipes technical properties on design solutions and structure safety. Purpose of the study is to analyze the influence of out-of-roundness of pipes and change of pipeline product transportation mode on design solutions and structure safety, give the design solution for provision of strength and safety. Object of study is pipeline with the section out-of-roundness under conditions of change of transportation mode. Methods of study are the theoretical studies of stressed-deformed state and safety of pipelines with out-ofroundness under conditions of change of transportation mode. Results are as follows: the authors analyzed the influence of pipes out-of-roundness and changes of transportation mode on design solutions and structures safety, offered the design solution for provision of strength and safety.
\end{abstract}

Keywords - pipeline; out-of-roundness of pipes; transportation mode; internal pressure; strength; safety.

\section{INTRODUCTION}

One of the main trends of provision of strength and safety of main oil and gas pipelines is making design solutions taking into account the actual technical properties of materials and items, actual operating conditions and adherence to the operating modes, which are built-in in the design solutions, during functioning. Strength and safety of the pipelines operation is formed by the level of mechanical stress in their metal and its change due to the unsteadiness of technological operating parameters. Level of mechanical stress depends on the operating loads and item structure perfection. Deviation of the pipe cross section from the round one under operating conditions leads to occurrence of additional stresses in pipe wall. Under conditions of technological operating parameters unsteadiness, the presence of pipes out-of-roundness is associated with the occurrence of the changing increased stresses. In this regard, it is required to evaluate strength and safety of oil and gas main pipelines under conditions of technological parameters unsteadiness taking into account outof-roundness of pipes.

Among oil and gas industry pipeline systems is the line section of the oil and gas main pipelines. Oil and gas main pipelines are characterized by a sufficiently high level of forces applied on them and stressed state, which are caused by the mode of their operation and its change, peculiarities of shape of pipes and their joints [1-7]. Pipes for construction of oil and gas main pipelines may have a section out-ofroundness as a manufacturing defect. In accordance with the requirements of the rule book [8], pipe ends out-of-roundness (ratio of the difference between maximum and minimum diameters in one section to nominal diameter) should not exceed one percent. Out-of-roundness of pipes with mall thickness of $20 \mathrm{~mm}$ and higher should not exceed 0.8 percent. Out-of-roundness requirements for the remaining pipe section are not determined.

\section{ON STUDY PROCEDURE}

Out-of-roundness of pipes makes it difficult to joint them by butt welding ensuring the regulatory requirements on edge displacement [9]. Edge displacement of butt joint pipes leads 
to the occurrence of additional stresses during operation, which decrease structure safety $[2,10]$. Moreover, in these cases if the internal pressure $p$ has impact in circumferential direction apart from hoop stress, which is determined via the formula $\sigma_{t}=\frac{p R}{\delta_{n}}$ (1), bending stresses appear. In the equation (1) $R, \delta_{n}$ mean radius and wall thickness of pipe.

Bending stresses, which occur under the influence of the inner pressure due to out-of-roundness of pipes cross section with $\mathrm{c}$ reduction of relation between the minimum and the maximum radii, increase significantly. E.g., when the ratio between the given radii deceases from 0.9 to 0.8 , up to 2.5 fold increase of bending stresses [11]. When the minimum and the maximum radii are equal (lack of out-of-roundness), bending stresses amount to zero. Analysis shows that the maximum hoop and bonding stresses occur in the wall of pipe with out-of-roundness in the section of its maximum diameter. Determination of stresses in the section of pipe minimum diameter is also of interest.

Taking into account the recommendation [12], bending stresses can be determined from the following analytical expression

$$
\sigma_{b i}= \pm \frac{6 p R_{2}^{2}}{\delta_{n}^{2}} \bar{M}_{i}
$$

being $\sigma_{b i}$ - bending stresses in pipe wall in the sections of maximum diameter $\left(\sigma_{b 1}\right)$ and minimum diameter $\left(\sigma_{b 2}\right)$;

$i$ - hereinafter amount to 1 or 2 , mean the correspondence of a parameter to the pipe section of maximum diameter $(i=1)$ and minimum diameter $(i=2)$;

$R_{1}$ - pipe maximum diameter;

$R_{2}$ - pipe minimum diameter;

$\bar{M}_{i}-$ dimensionless parameters of bending moment.

Dimensionless parameters of bending moment can be determined via the formulas obtained using the data given in the study [11]:

$$
\begin{gathered}
\bar{M}_{1}=0.2510\left[\left(\frac{R_{2}}{R_{1}}\right)^{-2.1603}-1\right], \\
\bar{M}_{2}=0.2675\left[\left(\frac{R_{2}}{R_{1}}\right)^{-1.7469}-1\right],
\end{gathered}
$$

Taking into account changes of pipe radius in cross section, hoop stresses will amount to

$$
\sigma_{t}=\frac{p R_{i}}{\delta_{n}}
$$

Total stresses in sections of maximum and minimum diameter, which include hoop stresses and bending stresses, are determined via the formula

$$
\sigma_{i}=\sigma_{t} \cdot \bar{\sigma}_{i}
$$

being

$$
\sigma_{t}=\frac{p R_{n}}{\delta_{n}}
$$

$\sigma_{i}-$ total stresses in circumferential direction correspondingly in sections of maximum and minimum diameter of pipes;

$\bar{\sigma}_{i}$ - dimensionless parameters determined depending on geometrical characteristics of pipe and its out-of-roundness;

$$
R_{n}-\text { standard radius of pipe. }
$$

Calculation formulas for calculating the maximum values $\bar{\sigma}_{1}$ and $\bar{\sigma}_{2}$ are as follows

$$
\begin{aligned}
& \bar{\sigma}_{1}=\frac{R_{1}}{R_{n}}\left(1+\frac{6 R_{2}^{2} \bar{M}_{1}}{R_{1} \delta_{n}}\right) ; \\
& \bar{\sigma}_{2}=\frac{R_{2}}{R_{n}}\left(1+\frac{6 R_{2} \bar{M}_{2}}{\delta_{n}}\right) .
\end{aligned}
$$

III. ANALYSIS OF INFLUENCE OF PIPES OUT-OF-ROUNDNESS AND INTERNAL PRESSURE ON LEVEL OF MECHANICAL STRESSES

Values of stresses in pipe walls, which occur in circumferential direction under the impact of the internal pressure, for the pipeline with the standard radius of $60.3 \mathrm{~cm}$ and the wall thickness of $1.4 \mathrm{~cm}$ are given in the Table 1 . Stresses are calculated for various values of internal pressure. Hoop stresses $\sigma_{t}$ are calculated via the formula (7) and occur in the absence of pipes out-of-roundness, i.e. if $R_{1}=R_{2}$. Stresses $\sigma_{1}$ are calculated taking into account pipes out-ofroundness and given that $\frac{R_{2}}{R_{1}}=0.99$ and $\frac{R_{1}}{R_{n}}=1.01$; they include hoop stresses, which are calculated via the formula (2), and hoop stresses, which are calculated via the formula (5) and which have impact in circumferential direction. 
TABLE I. - DEPENDENCES OF TOTAL $\left(\sigma_{1}\right)$ AND HOOP STRESSES $\left(\sigma_{t}\right)$ ON THE INTERNAL PRESSURE FOR PIPELINE WITH THE RADIUS OF $R_{n}=60.3 \mathrm{CM}$ AND THE WALL THICKNESS OF $\delta_{n}=1.4 \mathrm{CM}$

\begin{tabular}{|c|c|c|c|c|c|c|c|}
\hline $\begin{array}{l}p, \\
\mathrm{MPa}\end{array}$ & $\mathbf{2 . 0}$ & $\mathbf{2 . 5}$ & $\mathbf{3 . 0}$ & $\mathbf{3 . 5}$ & $\mathbf{4 . 0}$ & $\mathbf{4 . 5}$ & $\mathbf{5 . 0}$ \\
\hline $\begin{array}{c}\sigma_{t}, \\
\mathrm{MPa}\end{array}$ & 86.1 & 107.7 & 129.2 & 150.8 & 172.3 & 193.8 & 215.4 \\
\hline $\begin{array}{c}\sigma_{1}, \\
\mathrm{MPa}\end{array}$ & 198.8 & 248.4 & 298.1 & 346.7 & 397.5 & 445.8 & 495.3 \\
\hline
\end{tabular}

Analysis of calculation results given in the Table 1 shows that out-of-roundness leads to an up to 2.3-fold increase in stress levels. Increase in the pipe diameters leads to an increase in the influence of the out-of-roundness on the level of stresses. E.g., when $p=3.0 \mathrm{MPa}, \frac{R_{2}}{R_{1}}=0.975 ; \sigma_{t s}=550 \mathrm{MPa}$ for the pipe with the radius of $R_{n}=60.3 \mathrm{~cm}$ and the wall thickness of $\delta_{n}=1.4 \mathrm{~cm}$, stresses $\sigma_{1}$ are 1.2 times higher than for the pipe with the radius of $R_{n}=35.5 \mathrm{~cm}$ and the wall thickness of $1.0 \mathrm{~cm}$.

During transportation of oil and oil products, rapid change of transported fluid movement velocity occurs due to various reasons and, as a result, this leads to the local increase in the pressure by $\Delta p$ in the pipeline interior [13]. Local increase in pressure leads to local increase in pipe diameter, which leads to local longitudinal bending of wall [14]. The authors obtained the following analytical expression for determination of maximum bending stresses

$$
\sigma_{b i}=\sigma_{t} \cdot \bar{\sigma}_{b i}
$$

being

$$
\bar{\sigma}_{b i}=1.8157 \frac{\Delta p}{p} \bar{\sigma}_{i}
$$

$\sigma_{b i}$ - longitudinal bending stresses in the section of pipes maximum diameter;

$$
\bar{\sigma}_{b i}-\text { dimensionless parameter of the corresponding }
$$
stress.

Dimensionless parameter $\bar{\sigma}_{i}$ is calculated via the formulas (8) or (9).

Total maximum hoop stresses (stresses in circumferential direction) $\sigma_{t . c i}$ are calculated based on the following expression

$$
\sigma_{t . c .}=\sigma_{t} \cdot \bar{\sigma}_{t . c .}
$$

being $\quad \bar{\sigma}_{t . c .}=\left(1+1,5447 \frac{\Delta p}{p}\right) \bar{\sigma}_{i}$.

In the section of change of internal pressure, pipe wall is subject to the impact of stresses in circumferential and longitudinal directions at the same time, which is why, according to the Beltrami theory of failure, strength test should be performed with determination of maximum equivalent stresses via the formula

$$
\sigma_{e . m i}=\sigma_{t} \cdot \bar{\sigma}_{e . m i},
$$

being $\bar{\sigma}_{e . m i}=\left[\bar{\sigma}_{b i}^{2}+\bar{\sigma}_{t . c .}^{2}+\bar{\sigma}_{b i} \cdot \bar{\sigma}_{t . c i}\right]^{0.5}$.

Studies proved that, in order to reduce the local increase in pressure and reduce maximum stresses, it is necessary to reduce the change intensity of velocity of product movement in the pipeline interior over time. The problem of smooth change of the product movement velocity can be solved, e.g., with the help of main pumps equipped with a variablefrequency electric drive. Smooth change of the product movement velocity is provided by the smooth change of the pipeline internal pressure.

For the case of the smooth pressure change, parameters $\bar{\sigma}_{b 1}, \bar{\sigma}_{b 2}, \bar{\sigma}_{t . c 1}, \bar{\sigma}_{t . c 2}$ are calculated via the formulas

$$
\begin{gathered}
\bar{\sigma}_{b i}=1.8157 \frac{\Delta p}{p a_{0}} \bar{\sigma}_{i}, \\
\bar{\sigma}_{t . c .}=\left(1+1.5447 \frac{\Delta p}{p a_{0}}\right) \bar{\sigma}_{i} .
\end{gathered}
$$

Here $a_{0}=\beta \cdot l_{0}$, where $\beta$ is a parameter determined depending on the pipe geometrical characteristics and mechanical properties of its metal. E.g., for the steel pipe with the outer diameter of $102 \mathrm{~cm}$ and the wall thickness of $1.1 \mathrm{~cm}$, value $\beta=0.1725 \frac{1}{\mathrm{~cm}} ; l_{0}$ is the length of the part of the pipeline, where change of internal pressure from $p$ до $(p+\Delta p)$ occurs.

Analysis of the analytical expressions for stresses calculation given in the present articles shows that dependences of changes of the corresponding dimensionless parameters of stresses $\bar{\sigma}_{i}, \bar{\sigma}_{b i}, \bar{\sigma}_{t . c i}, \bar{\sigma}_{e . m i}$ on the pipes geometrical characteristics $R_{1}, R_{2}, \delta_{n}$ and parameters of loading $\frac{\Delta p}{p}, a_{0}$ fully relate to the stresses occurring in the pipeline. That is why the regularities of changes of stresses depending on the pipes geometrical characteristics and the parameters of loading $\frac{\Delta p}{p}$ and $\boldsymbol{a}_{(12)}$ gan be determined based 
on the analysis of the corresponding dimensionless parameters.

Level of safety factors is the important indicator of the structure safety. Increase in safety factor reduces intensity of emergency and risk [15]. Dependences of changes of safety factors on the parameters of loading, the pipes geometrical characteristics and the metal properties characterize the dependences of changes of the risk of emergency on the specified data. Safety factor based on the tensile strength $n_{t s}$ is taken within the range $1.7 \ldots 2.5$, and the one based on yield strength $n_{y}$ - within the range $1.2 \ldots 2.0$. For similarity of the analysis that will be applicable for all diameters and various steel grades of pipelines, the authors determined safety factors using the dimensionless complex parameters, which characterize the pipes geometrical characteristics $R_{n}, R_{1}, R_{2}, \delta_{n}$, mechanical properties of their metal and parameters of loading $p, \Delta p, \boldsymbol{a}_{O}$.

Thus, the authors have the following formulas for determination of safety factors

$$
\begin{gathered}
n_{t s j}=\frac{\bar{\sigma}_{t s}}{\bar{\sigma}_{j}}, \\
n_{y j}=\frac{\bar{\sigma}_{y}}{\bar{\sigma}_{j}},
\end{gathered}
$$

being $\quad \bar{\sigma}_{t s}=\frac{\sigma_{t s} \cdot \delta_{n}}{p \cdot R_{n}}$,

$$
\bar{\sigma}_{y}=\frac{\sigma_{y} \cdot \delta_{n}}{p \cdot R_{n}}
$$

In the expressions $(18) \div(21) \sigma_{t s}, \sigma_{y}$ are tensile strength and yield strength of pipes metal, and $\bar{\sigma}_{j}$ means $\bar{\sigma}_{1}, \bar{\sigma}_{2}$, $\bar{\sigma}_{b 1}, \bar{\sigma}_{b 2}, \bar{\sigma}_{t . c .}, \bar{\sigma}_{t . c .}, \bar{\sigma}_{e . m 1}$ and $\bar{\sigma}_{e . m 2}$.

In the Fig. 1 one can see the safety factors $n_{t s}$ and $n_{y}$ versus radiuses ratio $\frac{R_{2}}{R_{1}}$ curve, being $\frac{R_{1}}{R_{n}}=1.01$ for the pipeline with the standard radius of $R_{n}=60.3 \mathrm{~cm}$ and the wall thickness of $\sigma_{\mathrm{n}}=1.4 \mathrm{~cm}$. internal pressure $p=2.5 \mathrm{MPa}$. Tensile strength of pipes metal $\sigma_{t s}=550 \mathrm{MPa}$, yield strength $\sigma_{y}=450 \mathrm{MPa}$. Safety factors re determined via (18) and (19). Parameter $\bar{\sigma}_{j}=\bar{\sigma}_{e . m l}$, where $\bar{\sigma}_{e . m l}$ is calculated via (15).

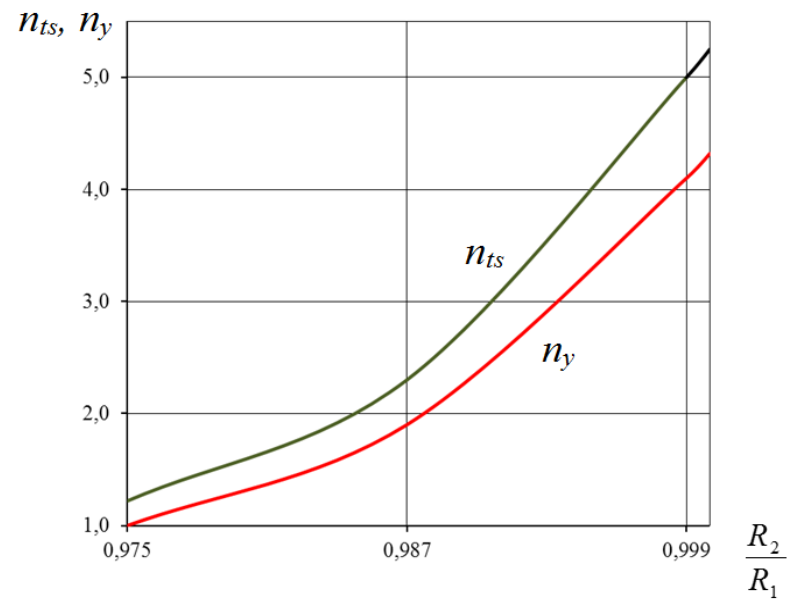

Fig. 1. Tensile strength $n_{t s}, n_{y}$ versus $\frac{R_{2}}{R_{1}}$ curves

As one can see from the given curves, decrease in $\frac{R_{2}}{R_{1}}$ is associated with a significant reduce of pipes safety factors and safety of main pipeline operation.

In the Fig. 2, one can see stresses parameters $\bar{\sigma}_{e . m 1}, \bar{\sigma}_{t . c 1}$ and $\bar{\sigma}_{b 1}$ versus $\frac{\Delta p}{p}$ curves, being $\frac{R_{2}}{R_{1}}=0.99 ; \frac{R_{1}}{R_{n}}=1.01$; $\frac{R_{2}}{\delta_{n}}=39.21$. Parameter $\bar{\sigma}_{b 1}$ is calculated via the formula (11), $\bar{\sigma}_{t . c .}-\operatorname{via}(13), \bar{\sigma}_{t . m 1}-\operatorname{via}(15)$.

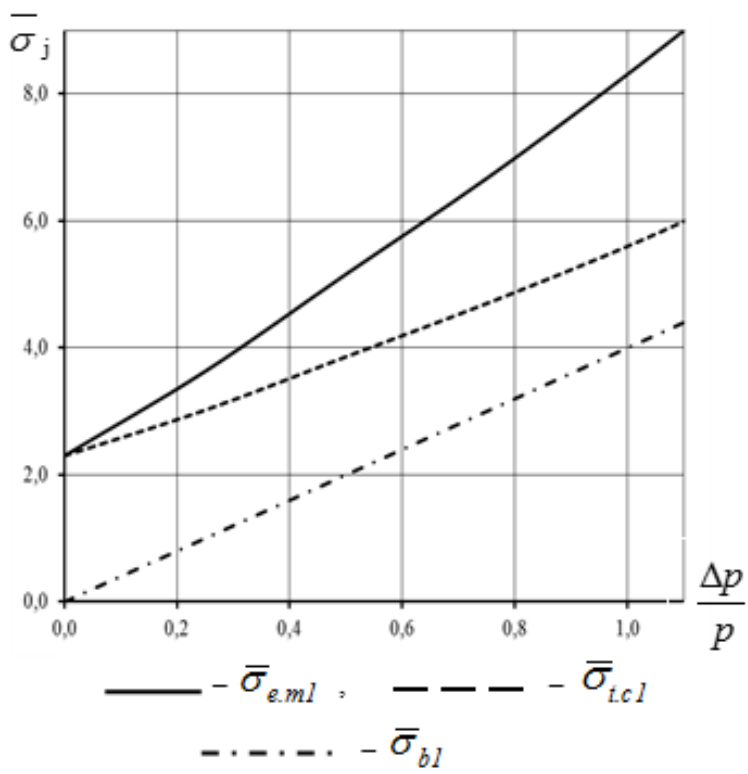

Fig. 2. Parameters $\bar{\sigma}_{e . m 1}, \bar{\sigma}_{t . c 1}, \bar{\sigma}_{b 1}$ versus $\frac{\Delta p}{p}$ curves 
For the comparative analysis, in Fig. 3 one can see dimensionless parameter of the maximum equivalent stress $\bar{\sigma}_{e . m}$ versus $\frac{\Delta p}{p}$ curve, being $R_{1}=R_{2}$, i.e. when there is no out-of-roundness. Comparison of the curves in the Fig. 2 and Fig. 3 shows that, for the analyzed condition, presence of outof-roundness leads to up to two-fold increase in the maximum equivalent pressure.

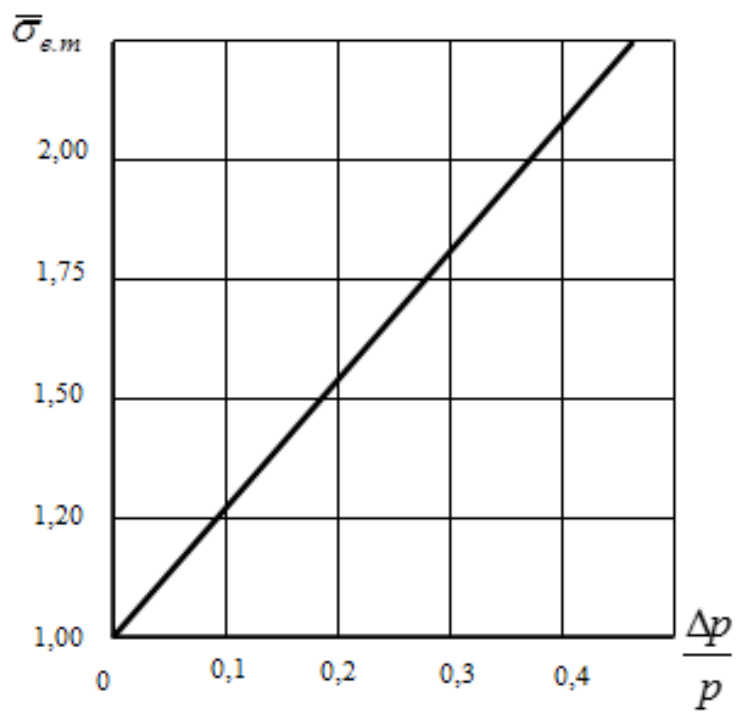

Fig. 3. Dimensionless parameter of the maximum equivalent stress versus change of pressure in the pipeline curve, being $R_{1}=R_{2}$

Local increase in internal pressure significantly increases the level of stresses in the pipelines with out-of-roundness. E.g., increase in $\frac{\Delta p}{p}$ from 0.25 to 0.75 leads to the 3.0 -fold increase in local bending stresses, 1.57 -fold increase in total hop stresses and 1.84-fold increase in maximum equivalent stresses in pipe walls.

\section{DESIGN SOLUTION FOR PROVISION OF STRENGTH AND SAFETY OF PIPELINE OPERATION}

Values of the parameter $\bar{\sigma}_{e . m 1}$, of safety factors $n_{t s}$ and $n_{y}$, being $\frac{\Delta p}{p}=0.5 ; \bar{\sigma}_{t s}=5.2 ; \bar{\sigma}_{y}=4.2$ and various $a_{O}$ are given in the Table 2 .

TABLE II. - DEPENDENCES OF $\bar{\sigma}_{e . m 1}, n_{t s}, n_{y}$ on $\boldsymbol{a}_{\boldsymbol{o}}$

\begin{tabular}{|c|c|c|c|c|c|c|}
\hline $\boldsymbol{a}_{O}$ & $\mathbf{0}$ & $\mathbf{2 . 5}$ & $\mathbf{5 . 0}$ & $\mathbf{1 0 . 0}$ & $\mathbf{2 0 . 0}$ & $\mathbf{3 0 . 0}$ \\
\hline $\bar{\sigma}_{e . m 1}$ & 5.16 & 3.36 & 2.74 & 2.45 & 2.33 & 2.28 \\
\hline$n_{t s}$ & 1.01 & 1.43 & 1.90 & 2.12 & 2.23 & 2.28 \\
\hline$n_{y}$ & - & 1.16 & 1.53 & 1.71 & 1.80 & 1.84 \\
\hline
\end{tabular}

Values of the dimensionless parameters of the maximum equivalent pressure $\bar{\sigma}_{e . m}$ and safety factors $n_{t s}$ and $n_{y}$, being $R_{1}=R_{2}$, various $a_{O}$ and the same values $\frac{\Delta p}{p}=0,5$; $\bar{\sigma}_{t s}=5,2 ; \bar{\sigma}_{y}=4,2$ are given in the Table 3 for comparative analysis with the data given in Table 2.

TABLE III. - DEPENDENCES OF $\bar{\sigma}_{e . m}, n_{t s}, n_{y}$ FROM $\boldsymbol{a}_{\boldsymbol{O}}$, BEING $R_{1}=R_{2}$

\begin{tabular}{|c|c|c|c|c|c|c|}
\hline $\boldsymbol{a}_{\boldsymbol{O}}$ & $\mathbf{0}$ & $\mathbf{2 . 5}$ & $\mathbf{5 . 0}$ & $\mathbf{1 0 . 0}$ & $\mathbf{2 0 . 0}$ & $\mathbf{3 0 . 0}$ \\
\hline $\bar{\sigma}_{e . m}$ & 2.36 & 1.82 & 1.65 & 1.57 & 1.54 & 1.52 \\
\hline$n_{t s}$ & 2.20 & 2.86 & 3.15 & 3.31 & 3.38 & 3.42 \\
\hline$n_{y}$ & 1.78 & 2.31 & 2.55 & 2.68 & 2.73 & 2.76 \\
\hline
\end{tabular}

Increase in parameter $\boldsymbol{a}_{O}$, which characterizes smoothness of change of velocity of transported product movement in the pipeline interior, leads to a significant (up to two-fold) decrease in $\bar{\sigma}_{e . m}$ and increase in $n_{t s}$ and $n_{y}$.

Moreover, in cases of rapid changes of transportation mode (e.g., by introducing the pumping unit, which is not equipped with a variable-frequency electric drive) under conditions of pipes out-of-roundness, safety factors are lower than the acceptable level. If there is no out-of-roundness and under conditions of rapid change of transportation mode, safety factors for the analyzed conditions are at the acceptable level and safety of pipeline systems operation is provided.

Oil transportation stops occur during operation due to various reasons. During the restart at the pipeline sections with pipes out-of-roundness, change of stresses in pipe walls from zero to the maximum values calculated via the formulas (6), (12) and (14) occurs. The authors evaluated the number of stress change cycles till breakage of pipes with out-ofroundness via Manson formula given in the study [16]. Calculations showed that increases in out-of-roundness significantly reduce the pipeline service line. E.g., for the pipe with standard radius of $R_{n}=60.3 \mathrm{~cm}$, wall thickness $\delta_{n}=1.4$ $\mathrm{cm}$, in case of change of internal pressure from zero to 4.0 $\mathrm{MPa}$ with increase in out-of-roundness, which is characterized by the decrease in $\frac{R_{2}}{R_{1}}$ from 0.975 to 0.950 in the absence of other pipes defects, number of cycles till breakage reduces down to 5,5-fold. The increase in the values of internal pressure $p$ and its local increase $\Delta p$ also reduces the service life of pipes with out-of-roundness. Smooth change of the pipeline capacity makes it possible to definitely preserve the service life. For the pipeline reviewed above, being 
$p=5.0 \mathrm{MPa}, \frac{\Delta p}{p}=0.5, \frac{R_{2}}{R_{1}}=0.99$, increase in parameter

$\boldsymbol{a}_{\boldsymbol{O}}$ from 10.0 to 20.0 is associated with up to a 1.3 -fold increase in pipes service life.

\section{CONCLUSION}

The authors noticed the high level of forces and occurring stresses influencing on the main oil and gas pipeline, which depend on the operation mode and peculiarities of pipes shape. Deviation of the pipe cross section from the round one leads to the occurrence of additional stresses in pipe walls during the process of product transportation. In the conditions of change of the transportation mode, pipes out-of-roundness is associated with the occurrence of variable elevated stresses. Theoretical studies of the stressed-deformed state and safety of pipeline under conditions of change of the transportation mode and pipes out-of-roundness were performed. The authors evaluated the analytical dependences of bending stresses and total hoop stresses, maximum equivalent stresses in the pipe walls on their geometrical characteristics, parameters of loading and changes of these parameters of loading under conditions of change of transportation mode taking into account pipes out-of-roundness. It is established that changes of transportation mode and pipes out-ofroundness lead to the increase in the level of the pipeline stressed state and the decrease in safety.

High-quality pipes manufacturing providing their out-ofroundness of not more than $0.8 \%$ along their full length, smooth design change of the product transportation using main pipeline pumping units equipped with a variablefrequency electric drive, make it possible to two-fold decrease the level of stresses, increase safety factor and service life, provide safe operation of oil and gas industry pipeline systems.

\section{References}

[1] R.S. Zainullin, "Increasing oil pipeline service life", Nedra, 494 p., 2000

[2] R.N. Bakhtizin, "Evaluation of stressed-deformed state of main pipeline pipes under conditions of change of transportation mode by turning on (turning off) main pipelines", Socar proceedings, No. 1, pp. 35-39, 2015 .

[3] Yu.V. Lisin, D.A. Neganov, N.A. Makhutov, N.E. Zorin, "Taking into account scale effect when justifying strength of main pipelines", Oil Industry, No. 6, pp. 112-116, 2017.

[4] Yu.V. Lisin, N.A. Makhutov, D.A. Neganov, V.M. Varshitsky, "Complex analysis of pipeline safety factors and basic mechanical prperties o pipe steels", Science and technologies of oil and oil products pipeline transport, No. 1 (28), pp. 30-38, 2017.

[5] R.S. Gasparyants, "Calculation for strength and durability of pipeline weld joints with defects [Text]", Oil Industry, No. 12, pp. 102-104, 2017.

[6] R.S. Gasparyants, "Calculation for strength and durability of pipelines with metal loss corrosion defects", Oilfield Engineering, VNIIOENG, No. 1, pp. 34-39, 2008.

[7] N.A. Makhutov, "Studies of strength of main pipelines with critical damages represented by corrugations [Text]", Labor Safety in Industry, No. 8, pp. 51-60, 2013.

[8] SP 36.13330.2012. Main Pipelines. M., 2012. 93 p.

[9] SP 86.13330.2012. Main Pipelines. M., 2012. 51 p.

[10] Kh.A. Azmetov, Z.Kh Pavlova, "Provision of safety of main pipelines operation under conditions of change of transportation mode", Scientific and Technical Journal "Environment Protection in Oil and Gas Complex", No. 5, pp. 37-40, 2014.

[11] G.K. Klein, "Calculation of underground pipelines", House for Literature on Construction, 240 p., 1969.

[12] S.P. Timoshenko, S. Voinovsky-Kriger, "Plates and sheaths", Nauka., 635 p., 1966.

[13] B.V. Ferichenkova, "Protection of main pipelines from hydraulic impact phenomena by on-coming discharge waves" Izvestiya of Higher Educational Institutions "Oil and Gas", No 1, pp. 42-48, 2009.

[14] V.I. Fedoseev, "Strength of materials", Nauka, 544 p., 1970

[15] N.A. Makhutov, V.N. Permyakov, "Safe service life of vessels and pipelines", Publishing House, Nauka, 516 p., 2005.

[16] I.A. Birger, R.R. Mavlyutov, "Strength of Materials", Nedra, 560 p., 1986. 